\title{
Energy Transfer Between Rare Earth-doped Ceramic Nanoparticles for Gauging Strain and Temperature in Elastic Polymers
}

\author{
Gil Yeroslavsky $^{1}$, Kyohei Okubo $^{1,2^{*}}$, Masakazu Umezawa ${ }^{1,2}$, Karina Nigoghossian $^{2}$, \\ Doan Thi Kim Dung ${ }^{3,4}$, Keiji Miyata ${ }^{2}$, Koki Nomura ${ }^{2}$, Masao Kamimura ${ }^{1,2}$, and \\ Kohei Soga ${ }^{1,2,3}$ \\ ${ }^{1}$ Imaging Frontier Center (IFC), Research Institute for Science and Technology (RIST), \\ Tokyo University of Science, 2641 Yamazaki, Noda, Chiba 278-8510, Japan \\ ${ }^{2}$ Department of Materials Science and Technology, Tokyo University of Science, \\ 6-3-1 Niijuku, Katsushika-ku, Tokyo 125-8585, Japan \\ ${ }^{3}$ Research Institute for Biomedical Science, Tokyo University of Science, 2669 Yamazaki, \\ Noda, Chiba 278-0022, Japan \\ ${ }^{4}$ Division of Functional Imaging, Exploratory Oncology Research \& Clinical Trial \\ Center, National Cancer Center, 6-5-1 Kashiwanoha, Kashiwa 277-8577, Japan \\ *kyohei.okubo@rs.tus.ac.jp
}

\begin{abstract}
The field of self-reporting materials is gaining increased attention recently. These materials can convey the forces applied to them without the need to integrate external devices. The ability to report strain exerted onto polymers is especially important, as it might give indication of high levels of force that may damage the material. These materials could also be applied to in-dwelling medical devices such as artificial tendons and heart valves. In this work, we designed a self-reporting system based on energy transfer (ET) between rare earthdoped ceramic nanoparticles (RED-CNPs). When external strain and heat are applied, the efficiency of the ET changes, resulting in changes in the emission spectra. We chose particles that are excited by $808 \mathrm{~nm}$ radiation and emit in the over-thousand-nanometer near infrared, since this range offers high penetration to biological tissues. Nanoparticles (NPs) were synthesized using the thermal decomposition method and then extensively characterized. Xray diffraction analysis revealed that the doped $\mathrm{NaYF}_{4}$ crystals are in the $\beta$-phase, while inductively coupled plasma emission spectrometry demonstrated the existence of two types of NPs: $\mathrm{NaYF}_{4}: \mathrm{Nd}^{3+}, \mathrm{Yb}^{3+}$ and $\mathrm{NaYF}_{4}: \mathrm{Er}^{3+}$. The absorption and emission of the particles showed no ET between the two kinds of NPs when simply mixed together. In order to reduce the interparticle distance and allow ET, RED-CNPs were coated with polydopamine (PDA) and then conjugated with a polymeric linker. Scanning electron microscopy, dynamic light scattering and absorption analysis showed the successful coating with PDA and the creation of interconnected NP networks. Emission from the conjugated system showed evidence of $\mathrm{ET}$, while changes in the emission lifetime of $\mathrm{Yb}$ as measured by time-gated imaging further suggested ET between $\mathrm{Yb}$ and Er. Finally, the conjugated system was integrated into poly(dimethylsiloxane) and it was shown that as strain or heat are exerted onto it, the emission spectra under $808 \mathrm{~nm}$ excitation is varied.
\end{abstract}

Keywords: NIR, Energy Transfer, Self-reporting materials, RED-CNP

\section{Introduction}

The ability to monitor strain in various materials is of great importance. Over the past decades, a variety of methods to detect strain have been developed, including mechanical, electrical and optical ones [1]. For example, the integration of flexible fiber optic sensors into polymers is used in biomedical applications where qualities of light 
travelling in the fiber, such as intensity, phase and wavelength, are modified in response to forces applied to the polymer [2]. The disadvantage of these techniques is that they require incorporation of physical components into the polymer, which could vary the material's physical properties. However, new approaches for strain-evaluation that involve incorporation of luminescent agents into the polymer are being developed. By observing the changes in parameters such as luminescent intensity, lifetime and wavelength, it is possible to assess the strain that is applied to the host material. Some examples of these mechanocromatic systems include the use of aggregates of dye molecules that change their emission wavelength when mechanical force separates the aggregates into monomers. Rossi et al. conjugated pyrene molecules to poly(hydromethylsiloxane) and integrated them into a poly(dimethylsiloxane) (PDMS) elastomer [3]. Upon stretching, a decrease in the aggregate/monomer intensity ratio was detected. Another approach that might be employed to assess strain is by using Förster resonance energy transfer (FRET). This optical phenomenon occurs when an excited fluorophore ("donor") transfers its energy to an adjacent fluorophore ("acceptor"), which can then be emitted as a photon of a longer wavelength [4]. This energy transfer (ET) is non-radiative and occurs through dipole-dipole coupling, meaning its efficiency is proportional to the inverse of the sixth power of the distance between the two molecules. Radiative ET may also occur when distances are larger than a few $\mathrm{nm}$ and the efficiency in that case is inversely proportional to the second power of the distance. For example, Karthikeyan and Sijbesma have used FRET to detect strain in thermoplastic elastomers [5]. They conjugated donor and acceptor molecules to a polymer that was incorporated into the elastomer. In resting state, the fluorophores were sufficiently close to each other and upon excitation, emission from both species was detected. However, when the polymer was stretched, the distance between the fluorophores increased and an increase in the donor/acceptor emission was detected. We have recently used a much simpler methodology to report strain by ET and incorporated fluorescein and rhodamine $\mathrm{B}$ into PDMS by simple mixing and curing [6]. Moreover, we were able to create a 2dimensional strain map by using a simple digital camera and image processing software.

When designing optical materials for use in indwelling medical devices, one has to take into account the absorption and scattering of body tissues. While the current generation of mechanocromatic systems uses almost exclusively visible light, this range of electromagnetic radiation has very low penetration of living organisms [7]. On the other hand, near infrared (NIR) light has a much higher penetration of biological tissues and is recently gaining increased attention for use in bioimaging $[8,9]$. However, while organic dyes are the major NIR agents used for bioimaging, they suffer from several limitations such as rapid photodegradation and short luminescence lifetime [10]. One of the promising NIR agents currently tested for various bio-applications is rare earth doped ceramic nanophosphors (RED-CNPs). These are ceramic nanocrystals such as $\mathrm{NaYF}_{4}$ and $\mathrm{LaPO}_{4}$ that are doped with lanthanide ions such as $\mathrm{Er}^{3+}$, $\mathrm{Yb}^{3+}, \mathrm{Nd}^{3+}, \mathrm{Tm}^{3+}$, etc. These NIR agents offer high stability, emission in various wavelengths and luminescence lifetime in the order of milliseconds [11]. Recently, Van de Haar et al. have demonstrated interparticle FRET (IFRET) between $\mathrm{LaPO}_{4} \mathrm{NPs}$ that were doped with either $\mathrm{Tb}$ or $\mathrm{Eu}$ [12]. When the two types of particles were $3 \mathrm{~nm}$ or less from each other, ET from $\mathrm{Tb}$ to $\mathrm{Eu}$ occurred, resulting in emission at $700 \mathrm{~nm}$.

In this work, we set out to use a system comprised of two types of RED-CNPs that can maintain ET that is affected by various stimuli, including strain and heat. Similar to our previous work, when the distance between donor and acceptor NP is small enough, ET is efficient [6]. However, when stretched this distance increases thus decreasing the ET efficiency and changing the emission spectrum. To keep the interparticle distance small enough to allow efficient ET, we first coated them with polydopamine (PDA) and then used this coating to conjugate the particles to a poly(ethylenimine) (PEI) linker (Scheme 1). PDA is a biomimetic polymer based on mussel adhesive protein used by these organisms to adhere to surfaces [13]. It was discovered that PDA can coat almost any surface or particle and this coating can later interact with molecules or other materials via covalent or noncovalent interactions. This ability to functionalize surfaces has been used extensively to endow different properties to these materials, such as antibacterial, antifouling and anti-cancer activity [14-16]. In the case of covalent bond-forming, PDA is reactive towards amine groups which are present in PEI. After particle conjugation, they were integrated into biocompatible PDMS and the ability of the system to respond to strain and heat was evaluated. 


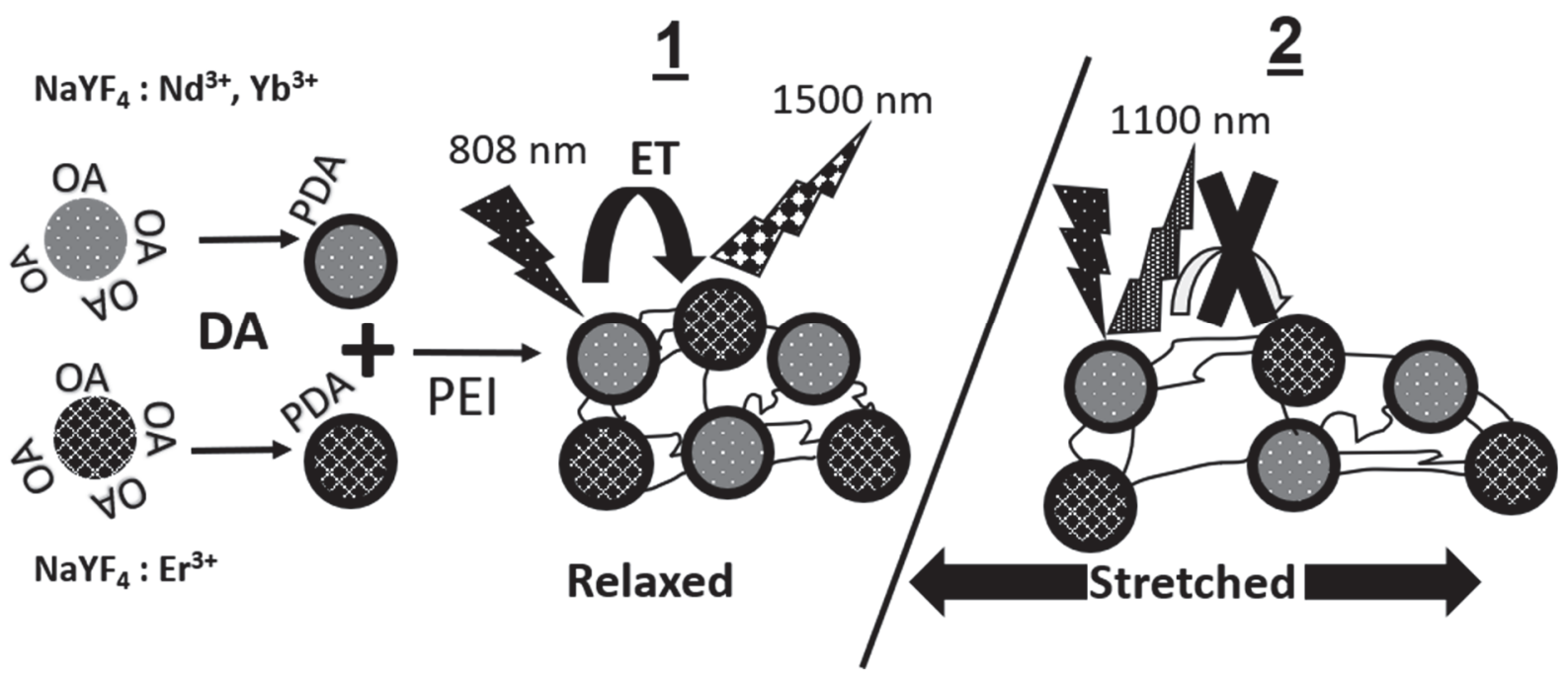

Scheme 1. Design of strain-responsive RED-CNP system. $\mathrm{NaYF}_{4} \mathrm{NPs}$ doped with $\mathrm{Nd}^{3+}$ and $\mathrm{Yb}^{3+}$ (grey) or $\mathrm{Er}^{3+}$ (black) and chelated by oleic acid (OA) were synthesized using the thermal decomposition method. After removal of OA, NPs were coated with a reactive PDA shell by simple mixing in Tris buffer containing dopamine (DA). The two types of PDA-coated NPs were then mixed together with PEI in Tris buffer to yield networks of PEI-conjugated RED-CNPs. In the relaxed state (1), NPs are close enough to each other to allow efficient interparticle ET. When irradiated with $808 \mathrm{~nm}$ light, $\mathrm{Nd}$ can absorb the energy and transfer it to $\mathrm{Yb}$ inside the particle, which can then be transferred to an adjacent $\mathrm{NaYF}_{4}: \mathrm{Er}^{3+}$ particle. This will be manifested by a strong emission from $\mathrm{Er}$ at $1500 \mathrm{~nm}$. However, when the system is stretched (2), the interparticle distance increases, resulting in decreased ET which should reduce the emission of Er, accompanied by a rise in $1100 \mathrm{~nm}$ emission from $\mathrm{Yb}$.

\section{Experimental}

\subsection{Materials}

All materials were purchased from Wako Chemicals (Japan) unless stated otherwise. Oleic acid (OA), yttrium chloride hexahydrate $\left(\mathrm{YCl}_{3} \cdot 6 \mathrm{H}_{2} \mathrm{O}\right)$, dopamine (DA) and nitrosyl tetrafluoroborate $\left(\mathrm{NOBF}_{4}\right)$ were purchased from SigmaAldrich (MO, USA).

\subsection{RED-CNP synthesis}

NPs were synthesized according to the thermal decomposition method [8]. Shortly, $30 \mathrm{~mL} 1$ octadecene (ODE) and $12 \mathrm{~mL} \mathrm{OA}$ were placed in a 3-necked flask with aqueous solutions of the different lanthanide chlorides. The quantities of the metals depended on the intended composition of each NP, but in all cases the combined amounts added up to $1 \mathrm{mmol}$. The flask was heated while being stirred and pumped with $\mathrm{N}_{2}$. Then $10 \mathrm{~mL}$ of $\mathrm{NaOH}$ and $\mathrm{NH}_{4} \mathrm{~F}$ (2.5 and $4 \mathrm{mmol}$, respectively) in methanol $(\mathrm{MeOH})$ was added and the mixture was heated to $300{ }^{\circ} \mathrm{C}$. Particles were then washcentrifuged (10 min, 30,000 rpm; 3-30 ks, Sigma, Germany) and suspended in hexane.

For PDA coating and PEI conjugation, particles were first stripped of their OA chelation and replaced with $\mathrm{BF}_{4}^{-}$. NPs in hexane $(5 \mathrm{mg} / \mathrm{mL})$ were mixed with $\mathrm{NOBF}_{4}(10 \mathrm{mg} / \mathrm{mL}$ in DMF $)$ overnight. After washing, the particles were suspended in water and reacted with dopamine (DA, 0.05 $\mathrm{mg} / \mathrm{mL})$ in $40 \mathrm{~mL}$ Tris buffer $(\mathrm{pH} 8.5,0.1 \mathrm{M})$ for 1 $\mathrm{h}$ to yield M@PDA particles. Particles were then washed in water and ethanol (EtOH). Particles were then suspended in $25 \mathrm{~mL}$ Tris buffer ( $\mathrm{pH} 9.5,0.1 \mathrm{M})$ and reacted with $100 \mu \mathrm{L} \mathrm{PEI}$ (branched, MW=1800) with overnight stirring at $40{ }^{\circ} \mathrm{C}$. Incorporation in PDMS was done by suspending the particles in 0.5 $\mathrm{mL}$ EtOH with $\mathrm{TiO}_{2}(0.01 \% \mathrm{w} / \mathrm{w})$, followed by mixture in the oligomer and crosslinker $(1: 0.1 \mathrm{~mL})$. The mixture was then degassed and cured at $65^{\circ} \mathrm{C}$ overnight to yield strips of $30 \times 10 \times 1(\mathrm{~L} \times \mathrm{W} \times \mathrm{H}) \mathrm{mm}$.

\subsection{Physical and optical characterization}

Samples were characterized by X-ray diffraction (XRD) using $\mathrm{CuK} \alpha$ radiation (RINT-TTR III, RIGAKU, Japan). The crystallite size $(D)$ of the particles was evaluated using the Scherrer equation (Eq. 1), where $K$ is the shape factor $(\sim 0.9), \lambda$ is the $\mathrm{X}$-ray wavelength (0.154), $\beta$ is the full width at half maximum (FWHM) and $\theta$ is Bragg's angle. [16]:

$$
D=\frac{K \lambda}{\beta \cos \theta}
$$


The peak chosen was at $43^{\circ}(201)$.

The morphology and size distribution were observed via scanning electron microscopy (SEM; HD-4200, Hitachi, Japan). The hydrodynamic diameter of the NPs was measured by a dynamic light scattering (DLS) analyzer (LB-550, Horiba, Japan).

Metal content was evaluated using inductively coupled plasma (ICP) emission spectrometry (ICPE-9000, Shimadzu Co., Kyoto, Japan). Samples were first digested in aqua regia $\left(\mathrm{HCl} / \mathrm{HNO}_{3}, 3: 1\right)$ overnight under stirring and then diluted in doubly distilled water (DDW). Quantification was done against multi-element calibration aqueous solutions: $\mathrm{Ce}, \mathrm{Dy}, \mathrm{Er}, \mathrm{Eu}, \mathrm{Gd}$, $\mathrm{Ho}, \mathrm{La}, \mathrm{Lu}, \mathrm{Nd}$, Pr, Sc, Sm, Tb, Th, Tm, Y, Yb (Perkin Elmer, CT, USA).

Absorption was measured with a V-770 spectrometer (Jasco, Japan). NIR Fluorescence was measured with Avantes spectrometer (NIR-256-1.7, Netherlands). Irradiation was done with $808 \mathrm{~nm}$ (normally at $1.5 \mathrm{~W} / \mathrm{cm}^{2}$ ) fiber-coupled laser diode (FL-FCSE08-7-808, Focus Light, China).

\subsection{Time-gated imaging and lifetime measurement}

The fluorescence lifetime was analyzed by timegated imaging (TGI) system, which is composed of pulsed laser and an NIR camera (ARTCAM0016TNIR; Artlay Co. Ltd., Tokyo, Japan) synchronized by a pulse generator (DG535; SRS Inc., Sunnyvale, CA, USA). A pulsed laser diode (wavelength: $808 \mathrm{~nm}$; power: $0.7 \mathrm{~W} / \mathrm{cm}^{2}$ ) was used to generate $1 \mathrm{~ms}$ pulses at a repetition rate of $20 \mathrm{~Hz}$. The pulse-to-pulse separation was set to $49 \mathrm{~ms}$, during which the fluorescence of the phosphors disappears completely. Two 850-nm long-pass filters (86-452; Edmund Optics, NJ, USA) were placed in front of the NIR camera. To obtain the fluorescence decay curves, a series of fluorescence images (8-bit) was acquired at delayed times ( $\left.t_{\text {delay }}\right)$ triggered by the pulse generator, where $t_{\text {delay }}$ ranged from 0 to $900 \mu \mathrm{s}$ in increments of $100 \mu \mathrm{s}$.

\subsection{Strain and temperature measurements}

PDMS-integrated samples were gradually stretched and irradiated with $808 \mathrm{~nm}$ laser $(2$ $\mathrm{W} / \mathrm{cm}^{2}$ ). Their emission spectra were measured with an NIR spectrometer. Similarly, PDMS strips were heated with a hot air gun and their spectra analyzed under 808 laser irradiation $\left(2 \mathrm{~W} / \mathrm{cm}^{2}\right)$.

\section{Results and discussion}

3.1. Particle synthesis and characterization
Three types of OA-chelated RED-CNPs were synthesized: $\quad \mathrm{NaYF}_{4}: \quad \mathrm{Nd}^{3+}{ }_{30 \%}, \quad \mathrm{Yb}^{3+}{ }_{10 \%}$ $(\mathrm{Nd} / \mathrm{Yb} @ \mathrm{OA}), \quad \mathrm{NaYF}_{4}: \quad \mathrm{Er}^{3+}{ }_{5 \%} \quad(\mathrm{Er} @ \mathrm{OA})$ and $\mathrm{NaYF}_{4}: \mathrm{Nd}^{3+}{ }_{30 \%}, \mathrm{Yb}^{3+}{ }_{10 \%}, \mathrm{Er}^{3+}{ }_{2 \%}(\mathrm{Nd} / \mathrm{Yb} / \mathrm{Er} @ \mathrm{OA})$ according to the thermal decomposition method [8]. The composition of $\mathrm{Nd} / \mathrm{Yb} @ \mathrm{OA}$ was designed according to the one used by Chihara et al., which ensures very bright emission from $\mathrm{Yb}$ [18]. In the aforementioned crystal lattice, $808 \mathrm{~nm}$ radiation is absorbed by $\mathrm{Nd}$ 's ${ }^{4} \mathrm{~F}_{5 / 2}$ energy level, followed by relaxation to ${ }^{4} \mathrm{~F}_{3 / 2}$, from which energy is transferred to the ${ }^{2} \mathrm{~F}_{5 / 2}$ level of $\mathrm{Yb}$ (energy level diagram is shown in Fig. S1) [19]. We optimized the Er content in Er@OA for maximum emission (Fig. S2), so that energy could be transferred from $\mathrm{Yb}$ to $\operatorname{Er}\left({ }^{2} \mathrm{~F}_{5 / 2} \rightarrow\right.$ $\left.{ }^{4} \mathrm{I}_{11 / 2}\right)$, followed by emission at around $1500 \mathrm{~nm}$. By comparing the $\mathrm{Yb}$ emission at $1100 \mathrm{~nm}$ to that of $\mathrm{Er}$ at $1500 \mathrm{~nm}$ we intend to infer the distance between the two particles. After washing and purification, NPs were suspended in hexane for physical characterization. First, the size of both particles was assessed using DLS and was found to be around 45 $\mathrm{nm}$ in diameter $(\mathrm{Nd} / \mathrm{Yb} @ \mathrm{OA}=43.0 \pm 9.9 \mathrm{~nm}$, $\mathrm{Er} @ \mathrm{OA}=46.7 \pm 9.5 \mathrm{~nm}$, Fig. 1A). Then particles were deposited on Si wafers for SEM analysis. As seen in Figure 1B, particles were fairly uniform in size and their average size was $43.5 \mathrm{~nm}$. XRD analysis was carried out on dry powders of the particles and demonstrated the crystals are in $\beta$ phase (reference pattern: JCPDS 16-0334, Fig. 2). The crystalline size was found to be about $11.5 \mathrm{~nm}$ in both cases. Particles were also analyzed for their metal content by ICP and their lanthanide composition was found to be almost identical to the theoretical one (Table S1).

The absorption spectra of the NPs are given in Fig. $3 \mathrm{~A}$, and for $\mathrm{Nd} / \mathrm{Yb} @ \mathrm{OA}$ many sharp absorption peaks can be seen, most of which are attributed to Nd. Most notable are the peaks at around $800 \mathrm{~nm}$
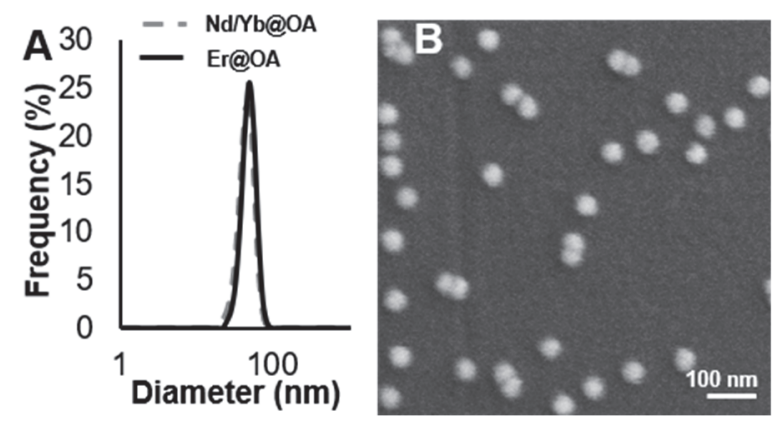

Fig. 1. Physical characterization of OA-chelated REDCNPs. A) Hydrodynamic diameter as determined by DLS. $\mathrm{X}$ axis is logarithmic. B) SEM image of $\mathrm{Nd} / \mathrm{Yb} @ \mathrm{OA}$ particles dispersed on a Si wafer. 


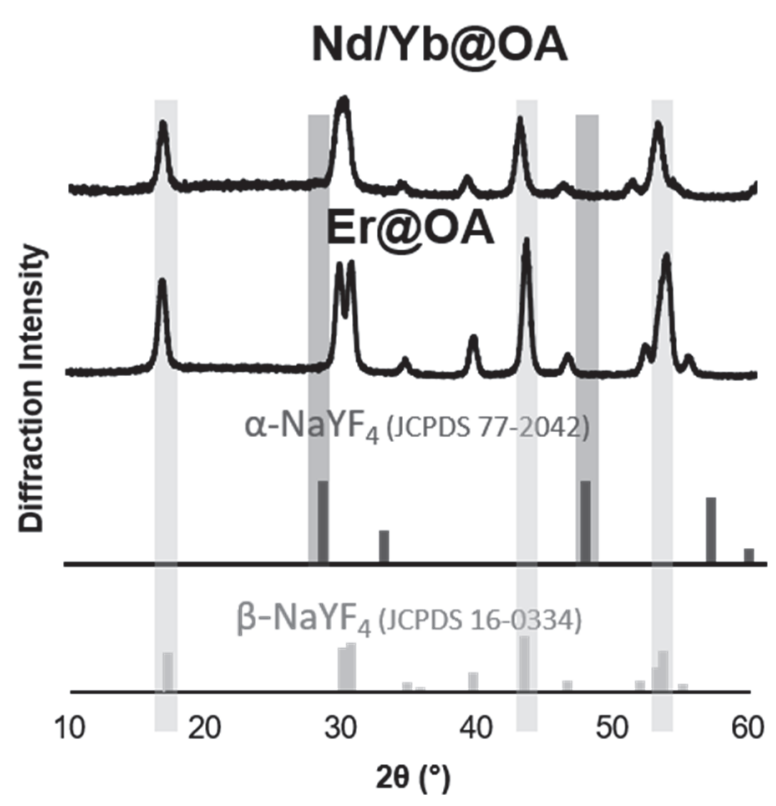

Fig. 2. XRD patterns for dried RED-CNPs.

$\left({ }^{4} \mathrm{I}_{9 / 2} \rightarrow{ }^{4} \mathrm{~F}_{5 / 2},{ }^{2} \mathrm{H}_{9 / 2}\right)$ and $600 \mathrm{~nm}\left({ }^{4} \mathrm{I}_{9 / 2} \rightarrow{ }^{4} \mathrm{G}_{5 / 2}\right.$, $\left.{ }^{2} \mathrm{G}_{7 / 2}\right)$. The absorption spectrum of Er@OA has far fewer sharp peaks, with a small one around $1000 \mathrm{~nm}$ $\left({ }^{4} \mathrm{I}_{15 / 2} \rightarrow{ }^{4} \mathrm{I}_{11 / 2}\right)$ [8]. Next, the emission of the particles suspended in hexane was determined and is shown in Fig. 3B. The emission at around 1100 $\mathrm{nm}$ is a combination of transitions ${ }^{4} \mathrm{~F}_{3 / 2} \rightarrow{ }^{4} \mathrm{I}_{11 / 2}$ and ${ }^{2} \mathrm{~F}_{5 / 2} \rightarrow{ }^{2} \mathrm{~F}_{7 / 2}$ from $\mathrm{Nd}^{3+}$ and $\mathrm{Yb}^{3+}$, respectively (Fig. S1) [20]. The peak at around $1300 \mathrm{~nm}$ corresponds to the transition ${ }^{4} \mathrm{~F}_{3 / 2} \rightarrow{ }^{4} \mathrm{I}_{13 / 2}$ from $\mathrm{Nd}^{3+}$. The emission of Er@OA presents one broad peak at around $1500 \mathrm{~nm}$, which is attributed to the ${ }^{4} \mathrm{I}_{13 / 2} \rightarrow$ ${ }^{4} \mathrm{I}_{15 / 2}$. This demonstrates the fact that $\mathrm{Er}$ can be weakly excited by $808 \mathrm{~nm}$ irradiation. Irradiation of a 1:1 mix of the two solutions showed no relative change in any of the peaks. If ET were to occur between $\mathrm{Yb}$ to Er, one would expect an increase in the Er peak and a decrease of the $\mathrm{Yb}$ one (1500 and $1100 \mathrm{~nm}$, respectively). Another good indication of ET would be a decrease in the 1100/1300 nm peak ratio. While the emission from $\mathrm{Yb}$ should be reduced due to ET to Er, the Nd peak should not be affected. To confirm this hypothesis, particles containing all three elements $(\mathrm{Nd} / \mathrm{Yb} / \mathrm{Er} @ \mathrm{OA})$ were prepared and their emission profile was compared to that of $\mathrm{Nd} / \mathrm{Yb} @ \mathrm{OA}$. Indeed, while the $1100 / 1300 \mathrm{~nm}$ peak ratio of $\mathrm{Nd} / \mathrm{Yb} @ \mathrm{OA}$ was 1.2 , co-doping the NPs with Er reduces the ratio to 0.6 (Fig. S3). However, in the case of a mixture of $\mathrm{Nd} / \mathrm{Yb} @ \mathrm{OA}$ and $\mathrm{Er} @ \mathrm{OA}$, that ratio remains unchanged. This demonstrates the fact that in a typical solution the distances between the two types of particles are too large for ET to occur.

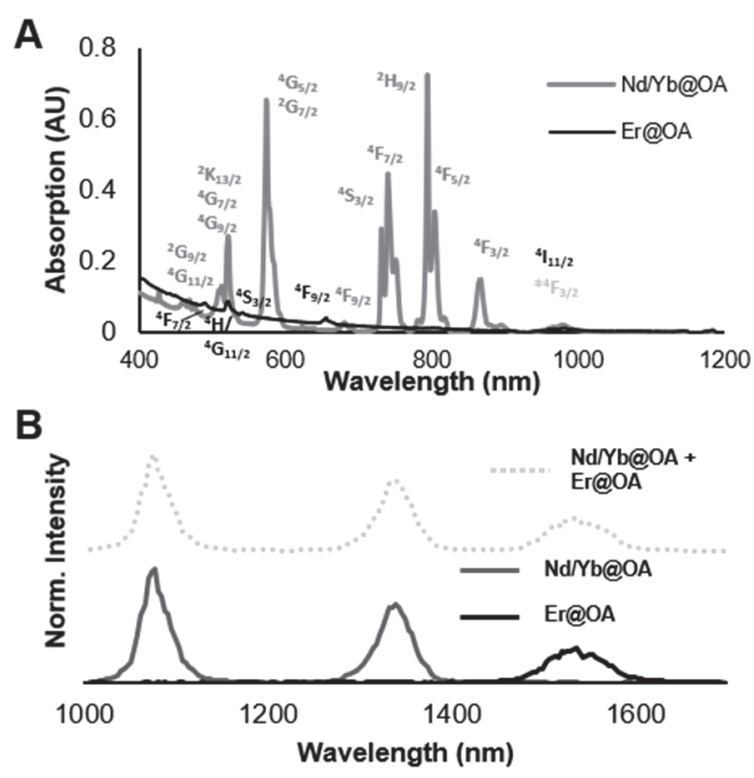

Fig. 3. Optical properties of OA-chelated RED-CNPs. A) Absorption spectra of the two types of particles with the postulated energy levels. The asterisk-marked energy level at $1000 \mathrm{~nm}$ is attributed to $\mathrm{Yb}$ in $\mathrm{Nd} / \mathrm{Yb} @ \mathrm{OA}$, while the grey ones belong to $\mathrm{Nd}$. Black energy levels are attributed to Er in Er@OA. B) Emission spectra of particles suspended in hexane. The bottom 2 spectra were taken separately for $\mathrm{Nd} / \mathrm{Yb} @ \mathrm{OA}$ and Er@OA, while the top one represents a 1:1 mix of the two. The $\mathrm{Yb} / \mathrm{Nd}$ emission in both cases remains unchanged at 1.1.

\subsection{Particle conjugation}

In order to decrease the interparticle distance, we set out to conjugate NPs with a polymeric linker. This was done by first coating each type of NP with a PDA shell, which is reactive towards amine functional groups [13]. This reactivity can later be used in order to conjugate it with a PEI linker, thus creating a network of interconnected RED-CNPs. PDA coating was done according to the method used by Liu et al. [21]. Shortly, each type of NP in hexane was mixed with a solution of $\mathrm{NOBF}_{4}$ in DMF overnight to yield particles chelated by $\mathrm{BF}_{4}$ $\left(\mathrm{M} @ \mathrm{BF}_{4}^{-}\right)$. Particles were then separately suspended in a DA solution in Tris buffer ( $\mathrm{pH} 8.5)$ for $1 \mathrm{~h}$ to yield PDA-coated RED-CNPs (M@PDA). First, DLS analysis was done on particles coated with PDA (Fig. 4A). While NPs chelated by OA were about $45 \mathrm{~nm}$ in size, the coated particles increased in size to about $50 \mathrm{~nm}$, indicating a shell of $2.5 \mathrm{~nm}$ thickness. In the procedure reported by Liu et al. a similar size was achieved, as measured by transmission electron microscopy (TEM). It should be noted that the difference in size herein was measured by DLS, which measures the hydrodynamic radius. For a hydrophilic polymer such as PDA, it is possible that a layer of water 

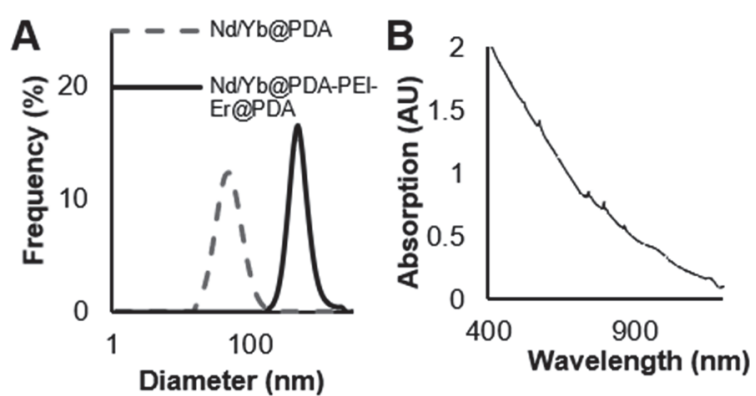

Fig. 4. Characterization of PEI-conjugated PDA-coated RED-CNPs. A) DLS studies of unconjugated and conjugated PDA-coated particles suspended in water. B) Absorption spectrum of Nd/Yb@PDA-PEI-Er@PDA in water.

around the polymer increases the measured size, while the actual size and shell thickness might be smaller. The absorption spectrum of coated NPs is given in Fig. 4B and the characteristic broad absorption spectrum of PDA can clearly be seen [13]. While most of the $\mathrm{Nd}$ and $\mathrm{Yb}$ peaks are covered by PDA's absorption, some peaks can still be seen, most notably around 600 and $800 \mathrm{~nm}$.

After confirmation of PDA coating, the two types of particles were reacted with PEI in Tris buffer $(\mathrm{pH}$ 9.5) overnight to give conjugated Nd/Yb@PDAPEI-Er@PDA. Examination of the DLS results in Fig. 4A show the reacted particles increased in size significantly. From $50 \mathrm{~nm}$, the average size grew to about $800 \mathrm{~nm}$. This is likely due to formation of big clusters of particles that are bound together by PEI. Further proof of this notion is given by SEM images shown in Fig. 5. Simply mixing the 2 types of PDAcoated NPs gives large plaques of material which are composed of NPs and PDA aggregates. The plaques are usually more than $10 \mu \mathrm{m}$ in size, although smaller clusters also appear. The PDA and RED-CNP parts can easily be distinguished by the different contrast. The polymeric parts are made of particles of about $50 \mathrm{~nm}$, which is typical for PDA (Fig. S4) [22]. The RED-CNPs are clustered together tightly, however their size is difficult to assess due to the merging of the PDA shells. $\mathrm{Nd} / \mathrm{Yb} @$ PDA-PEI-Er@PDA present a drastically different shape, characterized by round clusters of several hundreds of $\mathrm{nm}$. The particles are less packed together, probably due to the PEI spacer. A polymeric shell can clearly be seen around the REDCNPs, however no large PDA plaques are attached to the clusters. This difference in morphology between particles reacted with and without PEI demonstrates the successful covalent conjugation of the linker. Additional SEM images are given in Fig. S4.

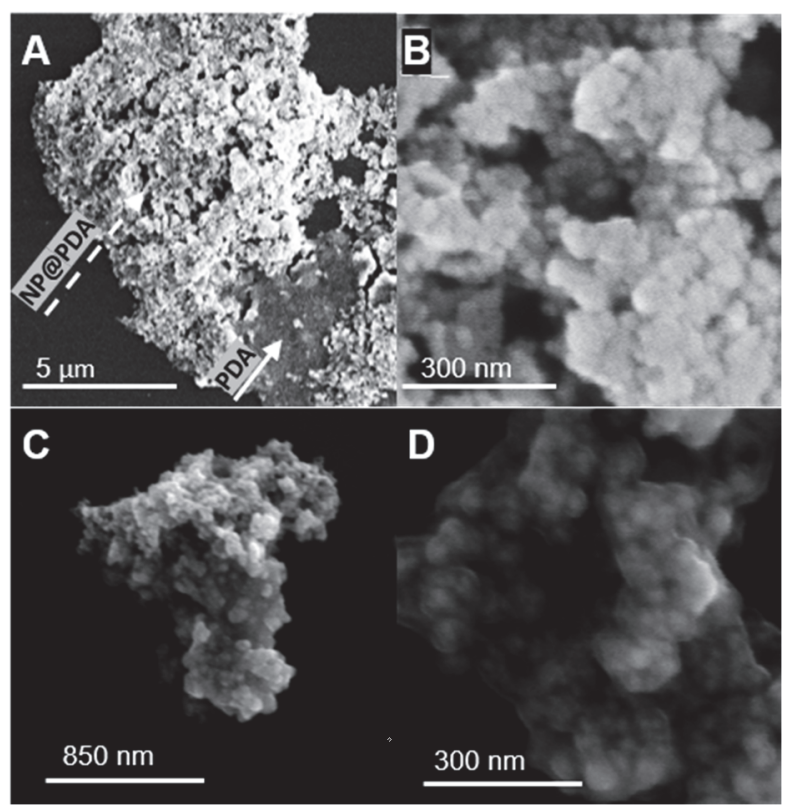

Fig. 5. SEM images of a mixture of $\mathrm{Nd} / \mathrm{Yb} @ \mathrm{PDA}$ and Er@PDA (A and B) and Nd/Yb@PDA-PEI-Er@PDA (C and D).

The PDA-coated particles were then dispersed in water and their emission spectra was evaluated under irradiation at $808 \mathrm{~nm}$. As seen in Fig. 6A, all particles lose some of their emission compared to their OA-chelated form, possibly because of PDA absorption or quenching by water. When comparing the separate PDA-coated particles

to their PEI-conjugated form, two differences are apparent. Firstly, for Er@PDA the emission at 1500 $\mathrm{nm}$ is very low, mostly because of Er's low absorption at $808 \mathrm{~nm}$. However, upon conjugation to $\mathrm{Nd} / \mathrm{Yb} @ \mathrm{PDA}$, this emission quadruples, giving a $\mathrm{Yb} / \mathrm{Er}$ emission ratio of 5. This is a big reduction from the ratio calculated for the emissions from separate $\mathrm{Nd} / \mathrm{Yb} @$ PDA and Er@PDA, which is 30. It should be noted that this increase cannot be attributed to reabsorption of emission from $\mathrm{Yb}$ and $\mathrm{Nd}$, since Er is not excited by radiation in 1100 and $1300 \mathrm{~nm}$. Secondly, the $\mathrm{Yb} / \mathrm{Nd}$ emission ratio for $\mathrm{Nd} / \mathrm{Yb} @ \mathrm{PDA}$ is reduced from 1.6 to 1.1 , in the conjugated form. This is not a dramatic decrease such as in the case of $\mathrm{Nd} / \mathrm{Yb} / \mathrm{Er} @ \mathrm{OA}$, but in the latter case the distance between the lanthanides is much smaller, which allows for a far more efficient ET. These two features of the water dispersed Nd/Yb@PDA-PEI-Er@PDA demonstrate that interparticle ET probably occurs between $\mathrm{Yb}$ and $\mathrm{Er}$.

Another evidence of ET between $\mathrm{Yb}$ and Er can be obtained by observing change in the emission lifetime of $\mathrm{Yb}$ with or without $\mathrm{Er}$ [19]. To that end, samples were dried on glass slides, 

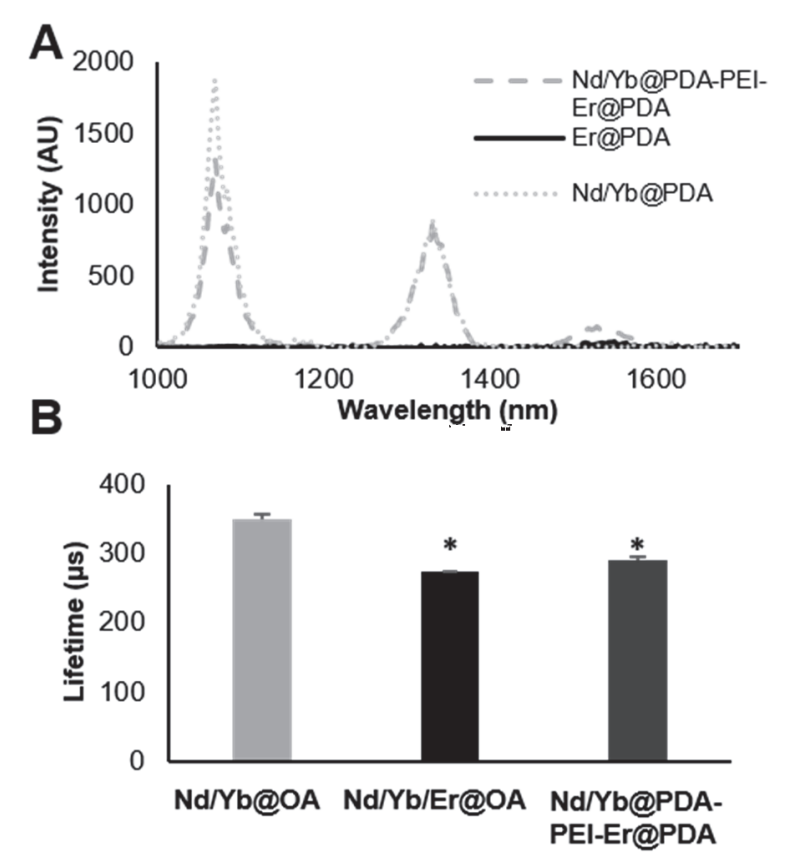

Fig. 6. Evidence of ET in PEI-conjugated PDA-coated RED-CNPs. A) Emission spectra in water of different particles treated with PDA. B) The decay lifetime of different particles during 0-300 $\mu \mathrm{s} . *$ denotes significant difference from $\mathrm{Nd} / \mathrm{Yb} @ \mathrm{OA}(\mathrm{p}<0.01)$. There was no significant difference between $\mathrm{Nb} / \mathrm{Yb} / \mathrm{Er} @ \mathrm{OA}$ and Nd/Yb@PDA-PEI-Er@PDA ( $>$ > 0.05), N=3.

irradiated with $808 \mathrm{~nm}$ pulse laser and their emissions analyzed with an NIR camera. First, the lifetime of $\mathrm{Yb}$ in $\mathrm{Nd} / \mathrm{Yb} @ \mathrm{OA}$ was compared to that of $\mathrm{Nd} / \mathrm{Yb} / \mathrm{Er} @ \mathrm{OA}$ (Fig. S5). It was assumed that the presence of Er would shorten the emission lifetime of $\mathrm{Yb}$ due to ET. Indeed, plotting the ln of emission from $\mathrm{Nd} / \mathrm{Yb} @ O A$ reveals a linear decrease, with a lifetime of $330 \mu \mathrm{s}$. However, the decay in the emission from $\mathrm{Nd} / \mathrm{Yb} / \mathrm{Er} @ \mathrm{OA}$ is much more complex, presenting 3 distinct decay patterns: from 0 and $300 \mu \mathrm{s}, 400$ to $600 \mu \mathrm{s}$, and then until $900 \mu \mathrm{s}$. The first calculated lifetime is $270 \mu \mathrm{s}$, followed by a very short lifetime of $20 \mu \mathrm{s}$ and then a slow one of about $400 \mu \mathrm{s}$. This intricate pattern is too complex for the scope of this work, however suffice to say it probably stems from the presence of Er. It's interesting to note that the second phase, which is probably the ET to Er, happens after $300 \mu$ s. This indicates that $\mathrm{Nd}$ 's ${ }^{4} \mathrm{~F}_{3 / 2}$ energy level first needs to fill, transfer its energy to $\mathrm{Yb}^{\prime}{ }^{2} \mathrm{~F}_{5 / 2}$ level and only then ET to Er can occur (Fig. S1). It is possible that the last period stems mostly from emission of Er and not $\mathrm{Yb}$. In any case, this behavior demonstrates the contribution of Er to the relaxation regime of $\mathrm{Yb}$. Moreover, for most of the recorded period, the lifetime is drastically reduced compared to $\mathrm{Yb}$ with $\mathrm{Nd}$ alone. When observing the emission decay of
Nd/Yb@PDA-PEI-Er@PDA, again a non-linear pattern emerges. Between 0 and $300 \mu$ s the lifetime is $290 \mu \mathrm{s}$, which is very similar to the same period measured for $\mathrm{Nd} / \mathrm{Yb} / \mathrm{Er} @ \mathrm{OA}$. The rest of the decay is characterized by a very long lifetime, of about 920 $\mu \mathrm{s}$. This slow decay might be due to the chelation of Er by PDA, which stabilizes the excited state and slows the emission from the system. The decay rate in the periods of $0-300 \mu \mathrm{s}$ is given in Fig. $6 \mathrm{~B}$ and it can be seen that while it is very similar for particles with Er, it is significantly longer for $\mathrm{Nd} / \mathrm{Yb} @ \mathrm{OA}$ alone. This is a potent demonstration that interparticle ET in Nd/Yb@PDA-PEI-Er@PDA reduces the lifetime of $\mathrm{Yb}$, in a way similar to that of intraparticle $\mathrm{ET}$ in the $\mathrm{Nd} / \mathrm{Yb} / \mathrm{Er}$ system.

\subsection{Strain and temperature monitoring in PDMS}

Finally, the ability of the conjugated RED-CNPs system to serve as a sensor for various stimuli was tested. Nd/Yb@PDA-PEI-Er@PDA were incorporated into the biocompatible elastic polymer PDMS and the change in ET between $\mathrm{Yb}$ and Er as a function of applied strain was evaluated. It is hypothesized that any strain applied to the conjugated system would result in an increase in the distance between $\mathrm{Nd} / \mathrm{Yb} @ \mathrm{PDA}$ and $\mathrm{Er} @$ PDA, which would translate to a decrease in the ET efficiency. This change in efficiency could be detected by monitoring the emission ratio between $\mathrm{Yb}$ and Er.

To verify this hypothesis, strips of PDMS impregnated with Nd/Yb@PDA-PEI-Er@PDA were gradually stretched under illumination with $808 \mathrm{~nm}$ laser while their emission spectra were recorded. Figure 7A shows the change in $\mathrm{Yb} / \mathrm{Er}$ emission ratio as strain is applied to the PDMS strip. While all of the emission peaks are reduced due to stretching and the practical dilution of the system, the response of each element is different. Whereas the change in emissions at $30 \%$ strain from $\mathrm{Nd}$ and Er are $-40 \%$ and $-50 \%$, respectively, the change in $\mathrm{Yb}$ emission is only $-25 \%$. Indeed, it can be seen that as the elastomer is stretched, the emission of $\mathrm{Yb}$ increases compared to that of Er, indicating a reduction in ET efficiency. From a ratio of about 1 in the relaxed state, the ratio increases by $33 \%$ in the stretched state, giving a sensitivity of about 0.1 per $10 \%$ strain. This increase in the emission of $\mathrm{Yb}$ compared to Er demonstrates the potential use of the $\mathrm{Nd} / \mathrm{Yb} / \mathrm{Er}$ system for strain monitoring. It was also observed that the $\mathrm{Yb} / \mathrm{Nd}$ ratio increased in a similar manner. It should be noted that the current system is not reusable and its response is not reversible. This 


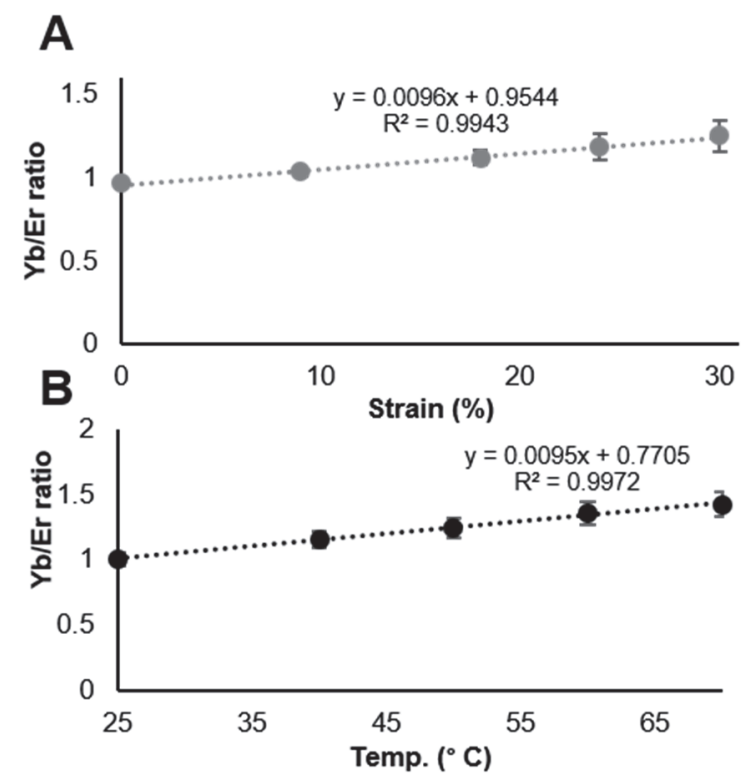

Fig 7. Response of $\mathrm{Nd} / \mathrm{Yb} @$ PDA-PEI-Er@PDA in PDMS to various stimuli. A) Effect of strain on $\mathrm{Yb} / \mathrm{Er}$ emission ratio. As the sample was stretched and irradiated with $808 \mathrm{~nm}$, the emission spectra were measured. B) Effect of temperature increase on $\mathrm{Yb} / \mathrm{Er}$ emission ratio.

might be due to corruption of the $\mathrm{Nd} / \mathrm{Yb} @ \mathrm{PDA}$ PEI-Er@PDA aggregates under strain, possibly because they are not flexible enough. In the current study branched PEI polymers of relatively low MW (1800) were used, which might lead to highly crosslinked clusters that are rigid and are prone to breakage. For that reason, optimization of the system is still needed.

Another possible use of this system is for temperature measurement, as the effect of heat on ET between $\mathrm{Yb}$ and $\mathrm{Nd}$ has already been reported [17]. In that work it was shown that as samples containing $\mathrm{NaYF}_{4}: \mathrm{Nd}^{3+}, \mathrm{Yb}^{3+}$ are heated, the luminescence lifetime of $\mathrm{Yb}$ is reduced due to phonon-assisted back transfer to $\mathrm{Nd}$. It stands to reason that if the lifetime and excitation energy of $\mathrm{Yb}$ are reduced, the ET between it and Er will be decreased, resulting in an increase of the $\mathrm{Yb} / \mathrm{Er}$ emission ratio. To test this assumption, PDMS samples with Nd/Yb@PDA-PEI-Er@PDA were heated and their emission spectra were recorded under $808 \mathrm{~nm}$ excitation. As seen in Fig. 7B, as temperatures increased, the ratio between the emission of $\mathrm{Yb}$ and Er was raised. As seen in Fig. S6, after an increase of $45^{\circ} \mathrm{C}$, the $\mathrm{Nd}$ peak increases by $60 \%$, while that of Er decreases by $25 \%$. This is consistent with energy back transfer from $\mathrm{Yb}$ to $\mathrm{Nd}$, which reduces the available energy for ET to Er. It's important to note the different behavior of $\mathrm{Nd}$ emission in the cases of either strain or heat application. While simply increasing the distance between NPs only raises the relative energy of $\mathrm{Yb}$ without any effect on $\mathrm{Nd}$, when heat is applied the effect is on both $\mathrm{Yb}$ and $\mathrm{Nd}$ due to phonon assistance. Overall, the linear change in $\mathrm{Yb} / \mathrm{Er}$ emission as the temperature increases shows that the current system might be suitable for deep tissue temperature monitoring, as NIR light can travel through several centimeters of biological tissues. This system may also be suitable for temperature sensing using TGI or emission lifetime, as those methods offer excellent sensitivity and are less affected by physiological conditions.

\section{Conclusion}

In this work we demonstrated the use of interparticle ET measurement as a means to gauge the strain and heat applied to elastomers. We have used two types of RED-CNPs: $\mathrm{NaYF}_{4}$ doped with $\mathrm{Nd}^{3+}$ and $\mathrm{Yb}^{3+}$ or with $\mathrm{Er}^{3+}$ due to their good absorption and emission in the NIR range. This range has high penetrability to biological tissues, which might enable the use of the system in indwelling biomedical devices. We showed that simply mixing the two particles does not produce efficient ET, as evident by very low emission from Er. However, as the particles are coated with a reactive PDA shell and conjugated together with a PEI linker, the interparticle distance decreases sufficiently. This allows for ET which is evident by a decrease in the emission from $\mathrm{Yb}$, accompanied by an increase from Er. Furthermore, luminescence lifetime measurements done with TGI revealed that the presence of Er in the system affects the emission from $\mathrm{Yb}$. Most importantly, attaching Er-containing $\mathrm{NPs}$ to $\mathrm{Nd} / \mathrm{Yb}$-containing NPs shortened the luminescence lifetime of $\mathrm{Yb}$ in a manner resembling that of NPs containing all three elements. Finally, we evaluated the ability of our system to serve as a sensor for various stimuli. After incorporation in PDMS, we demonstrated that strain or heat applied to the polymer changes the emission profile of the system. This shows the potential use of the current setup as a self-reporting material for various uses in the medical field.

\section{Acknowledgement}

This work was supported by the MEXTSupported Program for the Strategic Research Foundation at Private Universities \#S1511012. 


\section{References}

1. C. G'sell and J. J. Jonas, J. Mater. Sci., 14 (1979) 583.

2. P. Roriz, L. Carvalho, O. Frazão, J. L. Santos, and J. A. Simões, J. Biomech., 47 (2014) 1251.

3. N. A. A. Rossi, E. J. Duplock, J. Meegan, D. R. T. Roberts, J. J. Murphy, M. Patel, and S. J. Holder, J. Mater. Chem., 19 (2009) 7674.

4. T. Förster, Discuss. Faraday Soc., 27 (1959) 7.

5. S. Karthikeyan and R. P. Sijbesma, Macromolecules, 42 (2009) 5175.

6. G. Yeroslavsky, R. Inoue, M. Kamimura, Y. Kogo, and K. Soga, J. Photopolym. Sci. Technol., 31 (2018) 533.

7. A. M. Smith, M. C. Mancini, and S. Nie, Nat. Nanotechnol., 4 (2009) 710.

8. D. Jaque, C. Richard, B. Viana, K. Soga, X. Liu, and S. J. García, Adv. Opt. Photonics, 8 (2016) 1.

9. G. Yeroslavsky, M. Umezawa, K. Okubo, K. Nigoghossian, T. K. D. Doan, K. Miyata, M. Kamimura, and K. Soga, Biomater. Sci., (2020), https://doi.org/10.1039/C9BM02010A.

10. E. Hemmer, N. Venkatachalam, H. Hyodo, A. Hattori, Y. Ebina, H. Kishimoto, and K. Soga, Nanoscale, 5 (2013) 11339.

11. L. Wortmann, S. Suyari, T. Ube, M. Kamimura, and K. Soga, J. Lumin., 198 (2018) 236.

12. M. A. Van De Haar, A. C. Berends, M. R.
Krames, L. Chepyga, F. T. Rabouw, and A. Meijerink, J. Phys. Chem. Lett., 9 (2020) 51.

13. H. Lee, S. M. Dellatore, W. M. Miller, and P. B. Messersmith, Science, 318 (2007) 426.

14. G. Yeroslavsky, M. Richman, L. O. Dawidowicz, and S. Rahimipour, Chem. Commun., 49 (2013) 5721.

15. G. Yeroslavsky, R. Lavi, A. Alishaev, and S. Rahimipour, Langmuir, 32 (2016) 5201.

16. Z. H. Miao, H. Wang, H. Yang, Z. L. Li, L. Zhen, and C. Y. Xu, ACS Appl. Mater. Interfaces, 7 (2015) 16946.

17. J. I. Langford and A. J. C. Wilson, J. Appl. Crystallogr., 11 (1978) 102.

18. T. Chihara, M. Umezawa, K. Miyata, S. Sekiyama, N. Hosokawa, K. Okubo, M. Kamimura, and K. Soga, Sci. Rep., 9 (2019) 12806.

19. L. Tian, Z. Xu, S. Zhao, Y. Cui, Z. Liang, J. Zhang, and X. Xu, Materials, 7 (2014) 7289.

20. D. H. Ortgies, M. Tan, E. C. Ximendes, B. del Rosal, J. Hu, L. Xu, X. Wang, E. M. Rodríguez, C. Jacinto, N. Fernandez, G. Chen, and D. Jaque, ACS Nano, 12 (2018) 4362.

21. T. Liu, S. Li, Y. Liu, Q. Guo, L. Wang, D. Liu, and J. Zhou, J. Mater. Chem. B, 4 (2016) 2697.

22. G. Yeroslavsky, O. Girshevitz, J. Foster-Frey, D. M. Donovan, and S. Rahimipour, Langmuir, 31 (2015) 1064. 